\title{
BOUNDS ON THE MAXIMUM NUMBER OF VECTORS WITH GIVEN SCALAR PRODUCTS
}

\author{
M. DEZA AND P. FRANKL
}

\begin{abstract}
Suppose $M, L$ are sets of real numbers, $V=\left\{v_{1}, \ldots, v_{m}\right\}$ is a collection of vectors in $R^{n}$, having $k$ nonzero coordinates all from $M$ and satisfying $\left(v_{i}, v_{j}\right) \in L$ for $i \neq j$. Theorem 1.1 establishes a polynomial upper bound for $|V|$, generalizing previous results for subsets of a set and $(0, \pm 1)$-vectors. Theorem 1.4 asserts that if $|L|=s$ then $|V| \leqslant\left(\begin{array}{c}n+s \\ s\end{array}\right)$. For $M=\{-1,1\}, L=[-(k-1), k-1]$. Theorem 1.5 gives $|V| \leqslant 2^{k-1}\left(\begin{array}{c}n-1 \\ k\end{array}\right) / k$, where equality holds if and only if $V$ is a "signed" $(n, k, k-1)$ Steiner-system.
\end{abstract}

1. Introduction. Let $V=\left\{v_{1}, \ldots, v_{m}\right\}$ be a set of vectors in $R^{n}$, the $n$-dimensional Euclidean space. For $L, M$ subsets of real numbers, $0 \notin M$ and a positive integer $k$, we define $\mathscr{V}(n, k, M, L)$ as the collection of all $V$ satisfying the following three conditions:

(i) $v_{i}$ has exactly $k$ nonzero coordinates,

(ii) each nonzero coordinate of $v_{i}$ is from $M$,

(iii) $\left(v_{i}, v_{j}\right) \in L$ holds for $1 \leqslant i<j \leqslant m$.

The aim of this paper is to present upper bounds for the maximum size of $V$ with $V \in \mathscr{V}(n, k, M, L)$.

Let us denote this maximum by $m(n, k, M, L)$. Note that $m(n, k, M, L)$ can be infinite for some choices of the parameters. For $M=\{1\}$ this problem has been widely investigated (cf. e.g. [FrW]). The case $M=\{-1,+1\}$ was the subject of [DF1].

Define $M^{+}=\{m \in M: m>0\}, M^{-}=\{-m: m \in M, m<0\}$.

Let us also define $l_{\text {sup }}=\sup \{l: l \in L\}, m_{\text {inf }}=\inf \{|m|: m \in M\}$.

THEOREM 1.1. Suppose that $L^{+}$can be covered by $r$ intervals, each of length less than $m_{\mathrm{inf}}^{2} / 2$; then there exists a constant $c\left(k, l_{\text {sup }}, m_{\mathrm{inf}}\right)$ depending only on $k, l_{\text {sup }}$ and $m_{\mathrm{inf}}$ such that we have

$$
m(n, k, M, L) \leqslant c\left(k, l_{\text {sup }}, m_{\text {inf }}\right)\left(\begin{array}{l}
n \\
r
\end{array}\right) m\left(k, k, M^{+} \cup M^{-}, L^{+}\right) .
$$

The determination of $m(k, k, M, L)$ is a number theoretic question. It is easy to give examples for $m(k, k, M, L)=\infty$ (e.g. $L=Q=$ rationals $\},|M \cap Q|=\infty)$ or for $m(k, k, M, L)=1$ (e.g. $L=\{$ irrationals $\}, M \subset Q)$. Note that for $|M|$ finite

Received by the editors July 27, 1984 and, in revised form, November 14, 1984.

1980 Mathematics Subject Classification. Primary 05C35, 51M05; Secondary 51E99, 51K99. 
$m(k, k, M, L) \leqslant|M|^{k}$ holds. It is a consequence of Theorem 1 in [Fr] that $m(k, k, M,\{1\})<(2 k)^{2 k+1}$. We prove

Proposition 1.2. If $\left|L^{+}\right|$is finite then

$$
m(k, k, M, L) \leqslant(2 e)^{k}\left(\begin{array}{c}
\left|L^{+}\right|+k \\
k
\end{array}\right) .
$$

After preparations made in $\S 2$ we prove Theorem 1.1 in $\S 3$.

Let us note the following

COROLlaRY 1.3. If $\left|L^{+}\right| \leqslant r$ and $m_{\mathrm{inf}}>0$, then we have $m(n, k, M, L) \leqslant$ $c(k, L, M)\left(\begin{array}{l}n \\ r\end{array}\right)$.

Note that for $M=\{1\}$ the statement of the corollary (with $c(k, L, M)=1$ ) is a classical theorem of Ray-Chaudhuri and Wilson [RW].

For $|L|$ fixed we have the following more general bound:

TheOREM 1.4. Suppose $V=\left\{v_{1}, \ldots, v_{m}\right\}$ is a set of distinct vectors in $R^{n}$ so that, for each $v \in V$ and for a fixed positive integer $s,\left(v, v^{\prime}\right)$ takes up at most $s$ values (i.e., $\left.\left|\left\{\left(v, v^{\prime}\right): v \neq v^{\prime} \in V\right\}\right| \leqslant s\right)$. Then $|V| \leqslant\left(\begin{array}{c}n+s \\ s\end{array}\right)$.

Note the analogy with the following result of Bannai, Bannai, Stanton and Blokhuis. Recall that $S \subset R^{n}$ is called an $s$-distance set if $\mid\{d(x, y): x, y \in S$, $x \neq y\} \mid=s(d(x, y)$ is the euclidean distance of $x$ and $y)$.

THEOREM [BBS, B1]. If $S$ is an $s$-distance set in $R^{n}$, then $|S| \leqslant\left(\begin{array}{c}n+s \\ s-\end{array}\right)$.

Let us note also that if all vectors have equal length, say $b$, then $d(x, y)^{2}=(x-$ $y, x-y)=2 b-2(x, y)$. That is, the number of different distances and scalar products is the same. For this case there exists the stronger bound of Delsarte, Goethals and Seidel:

THEOREM [DGS]. If $S$ is an $s$-distance set on the unit sphere in $R^{n}$, then $|S| \leqslant\left(\begin{array}{c}n+s-1 \\ s\end{array}\right)$ $+\left(\begin{array}{c}n+s-2 \\ s-1\end{array}\right)$.

Recall that a $(n, k, t)$ partial Steiner-system $\zeta$ is a family of $k$-subsets of $\{1,2, \ldots, n\}$ such that every $t$-element subset is contained in at most one member of $\zeta$. Clearly $\zeta$ satisfies $|\zeta| \leqslant\left(\begin{array}{l}n \\ t\end{array}\right) /\left(\begin{array}{l}k \\ t\end{array}\right)(n \geqslant k>t \geqslant 1)$. In case of equality, $\zeta$ is called a Steiner-system. Very few Steiner-systems with $t>3$ and no Steiner-systems with $t \geqslant 6$ are known. However, recently Rödl [Rö] showed that for fixed $k, t$ and $n$ tending to infinity there exist partial Steiner-systems satisfying

$$
|\zeta| \geqslant(1-o(1))\left(\begin{array}{l}
n \\
t
\end{array}\right) /\left(\begin{array}{c}
k \\
t
\end{array}\right) .
$$

Given a partial Steiner-system $\mathscr{P}$ we may replace each $P \in \mathscr{P}$ by a collection of vectors $V_{P} \in \mathscr{V}(k, k,\{ \pm 1\},\{0, \pm 1, \ldots, \pm(t-1)\})$. Then $V_{\mathscr{P}}=\cup_{P \in \mathscr{P}} V_{P}: P \in \mathscr{P}$ is in $\mathscr{V}(n, k,\{ \pm 1\},[-(t-1), t-1])$. 
The maximum possible size of $V_{P}$ is a coding theoretical problem. Here we consider only the case $t=k-1$. Then the only restriction on $v, v^{\prime} \in V_{P}$ is that $v \neq-v^{\prime}$. Consequently, $\left|V_{P}\right| \leqslant 2^{k-1}$ and there are $2^{2^{k-1}}$ different ways to achieve equality here, namely choose exactly one out of each pair $\{v,-v\}$. If $\left|V_{P}\right|=2^{k-1}$ for all $P \in \mathscr{P}$, then $V_{\mathscr{P}}$ is called an antipodally signed partial Steiner-system.

THEOREM 1.5. Suppose $V \in \mathscr{V}(n, k,\{ \pm 1\},[-(t-1), t-1])$. Then

$$
|V| \leqslant \frac{\left(\begin{array}{l}
n \\
t
\end{array}\right)}{\left(\begin{array}{l}
k \\
t
\end{array}\right)} 2^{t-1}(k-t+1),
$$

moreover, for $k=t+1$, equality is possible if and only if $V=V_{\zeta}$ for some $(n, k, k-1)$ Steiner-system $\zeta$.

2. General reductions. For a vector $v=\left(v^{1}, v^{2}, \ldots, v^{n}\right)$ let us define the support $S(v)=\left\{i: v^{i} \neq 0,1 \leqslant i \leqslant n\right\}$.

LEMMA 2.1. For any set of vectors $V$ with $|S(v)|=k$ for all $v \in V$ there exists a partition $X_{1} \cup X_{2} \cup \cdots \cup X_{k}=\{1,2, \ldots, n\}$ such that the set $V^{\prime}=\{v \in V: \mid S(v)$ $\left.\cap X_{i} \mid=1, i=1, \ldots, k\right\}$ satisfies $\left|V^{\prime}\right| \geqslant\left(k ! / k^{k}\right)|V|$.

Proof. Let $X_{1} \cup \cdots \cup X_{k}$ be a random equipartition of $\{1,2, \ldots, n\}$. Thus $\left|X_{i}\right|$ $=\lfloor n / k\rfloor$ or $[n / k\rceil$. For a given $v \in V$ the probability of $\left|S(v) \cap X_{i}\right|=1$ for $i=1, \ldots, k$ is given by $\left|X_{1}\right| \cdots\left|X_{k}\right| /\left(\begin{array}{c}n \\ k\end{array}\right) \geqslant k ! / k^{k}$. Consequently, the expected number of members of $V^{\prime}$ is at least $\left(k ! / k^{k}\right)|V|$ and the statement follows.

Note that $k ! / k^{k}>e^{-k}$, thus by considering $V^{\prime}$, we only lose a constant factor. The family $V^{\prime}$ is called a transversal family of vectors.

LEMMA 2.2. To any transversal family $V^{\prime} \in \mathscr{V}(n, k, M, L)$ there exists $V^{\prime \prime} \in$ $\mathscr{V}\left(n, k, M^{+} \cup M^{-}, L^{+}\right)$satisfying $\left|V^{\prime \prime}\right| \geqslant 2^{-k}\left|V^{\prime}\right|$.

Proof. Associate with each $v \in V^{\prime}$ a $( \pm 1)$-vector of length $k$ by defining the $i$ th entry +1 if and only if the coordinate of $v$ in $S(v) \cap X_{i}$ is positive. There are $2^{k}$ different $( \pm 1)$-vectors of length $k$. Thus we may choose one, say $\left(\varepsilon_{1}, \varepsilon_{2}, \ldots, \varepsilon_{k}\right)$, which has been associated with at least $2^{-k}\left|V^{\prime}\right|$ vectors. Let $V^{*}$ be this collection and let $V^{\prime \prime}$ be the collection which we obtain from $V^{*}$ by applying the orthogonal transformation of multiplying the $i$ th coordinate of $v \in V^{*}$ by $\varepsilon_{i}, \quad V^{\prime \prime} \in$ $\mathscr{V}\left(n, k, M^{+} \cup M^{-}, L^{+}\right)$follows.

Note that Lemmas 2.1 and 2.2 imply

COROllary 2.3 .

$$
m(n, k, m, L) \leqslant(2 e)^{k} m\left(n, k, M^{+} \cup M^{-}, L^{+}\right) .
$$

Corollary 2.4.

$$
m(n, k, L) \leqslant m(n, k,\{ \pm 1\}, L) \leqslant(2 e)^{k} m\left(n, k, L^{+}\right) .
$$

For a set of vectors $V$ we define $\zeta(V)=\{S(v): v \in V\}$. 
Proposition 2.5.

$$
|V| \leqslant|\zeta(V)| m(k, k, M, L) .
$$

Suppose $V$ is a transversal family of vectors (for the partition $X_{1} \cup \cdots \cup X_{k}$ ). Then $\zeta(V)$ is a family of transversals. For a subset $H \subset X_{1} \cup \cdots \cup X_{k}$ we define the projection $\pi(H)$ of $H$ by $\pi(H)=\left\{i: H \cap X_{i} \neq \varnothing\right\}$.

Recall that a sunflower of size $t$ and with center $C$ is a collection $F_{1}, \ldots, F_{t}$ of sets with $F_{i} \cap F_{j}=C$ for $1 \leqslant i<j \leqslant t$.

A family of subsets $\mathscr{L}$ is called a meet semilattice if $L, L^{\prime} \in \mathscr{L}$ imply $L \cap L^{\prime} \in \mathscr{L}$. Also $\mathscr{I}(S, \zeta)=\left\{S \cap S^{\prime}: S^{\prime} \in \zeta\right\}$.

LemMa 2.6 (FÚREDI [Fü]). Given $k, t$, there exists a positive constant $c=c(k, t)$ such that any transversal family $\zeta$ of $k$-subsets contains a subfamily $\zeta^{*}$ satisfying:

(i) $\left|\zeta^{*}\right|>c(k, t)|\zeta|$,

(ii) every $C \in \mathscr{I}\left(S, \zeta^{*}\right)$ is the center of a sunflower of size $t\left(S \in \zeta^{*}\right)$,

(iii) $\mathscr{I}\left(S, \zeta^{*}\right)$ is a meet semilattice,

(iv) the family $\pi\left(\mathscr{I}\left(S, \zeta^{*}\right)\right)=\left\{\pi(C): C \in \mathscr{I}\left(S, \zeta^{*}\right)\right\}$ is independent of the choice of $S \in \zeta^{*}$.

For $\zeta=\zeta(V)$ let us apply Lemma 2.6 with $t=2$ and set $\mathscr{I}_{0}=\pi\left(\mathscr{I}\left(S, \zeta^{*}\right)\right)$.

A set $G \subset\{1,2, \ldots, k\}$ is called a generator set of $\mathscr{I}_{0}$ if $G \subset \mathscr{K} \in \mathscr{I}_{0}$ implies $\mathscr{K}=\{1,2, \ldots, k\}$.

Proposition 2.7. Suppose $\mathscr{I}_{0}$ has a generator set $G$ of size $r$. Then $\left|\zeta^{*}\right| \leqslant\left(\begin{array}{c}n \\ r\end{array}\right)$.

Proof. For each $S \in \zeta^{*}$ choose $G(S) \subset S$ so that $\pi(G(S))=G$ holds. Now $S^{\prime} \in \zeta^{*}, G(S) \subset S^{\prime}$ imply $G(S) \subseteq S \cap S^{\prime} \in \mathscr{I}\left(S, \zeta^{*}\right)$. Thus $S^{\prime}=S$ follows. Consequently, the $G(S)$ are all distinct $r$-subsets of $X_{1} \cup \cdots \cup X_{k}$. This yields $\left|\zeta^{*}\right| \leqslant\left(\begin{array}{c}n \\ r\end{array}\right)$.

Recall that a chain of length $r$ is a family $A_{0}, A_{1}, \ldots, A_{r}$ with $A_{0} \subset A_{1} \subset \cdots \subset A_{r}$.

Proposition 2.8. Suppose $\mathscr{I}_{0}$ contains no chain of length $r+1$. Then $\mathscr{I}_{0}$ has a generator set of size at most $r$.

Proof. Apply induction on $r$. If $r=1$ then $\mathscr{I}_{0}$ contains at most one proper subset $\mathscr{B}$ of $\{1,2, \ldots, k\}$. Thus $\{i\}$ is a generator for all $1 \leqslant i \leqslant k, i \notin \mathscr{B}$.

Suppose $\mathscr{B} \varsubsetneqq\{1,2, \ldots, k\}, \mathscr{B}$ is a maximal element in $\mathscr{I}_{0}$. Define $\tilde{\mathscr{I}}=\left\{\mathscr{B} \cap \mathscr{B}_{0}\right.$ : $\left.\mathscr{B}_{0} \in \mathscr{I}_{0}\right\}$. Then $\tilde{\mathscr{I}}$ has no chain of length $r$. Consequently, it has a generator set $H$ of size at most $r-1$. Now $G=H \cup\{i\}$ is a generator for $\mathscr{I}_{0}$ whenever $i \in$ $(\{1,2, \ldots, k\}-H)$.

3. The proof of Theorem 1.1. In view of Corollary 2.3 and Lemma 2.1 we may assume $V \in \mathscr{V}\left(n, k, M^{+} \cup M^{-}, L^{+}\right), V$ is a transversal family of vectors. By omitting at most $n$ vectors we may suppose no $v \in V$ has a coordinate position $v^{(i)}$ with $v^{(i)}>\sqrt{l_{\text {sup }}}$.

Let us set $\gamma=m_{\text {inf }}^{2} / 2 k \sqrt{l_{\text {sup }}}$. With $v \in V$, having positive coordinate $\alpha_{i}$ in $X_{i}$ we associate a sequence of integers $\left(t_{1}, \ldots, t_{k}\right)$ where $t_{i}=\left\lceil\alpha_{i} / \gamma\right\rceil$. 
This defines a partition of $V$ into at most $\left|\sqrt{l_{\text {sup }}} / \gamma\right|^{k}$ parts. This constant factor can be incorporated into $c\left(k, l_{\text {sup }}, m_{\text {inf }}\right)$, thus we may suppose: the same sequence $\left(t_{1}, \ldots, t_{k}\right)$ is associated with each $v \in V$.

In view of (2.1) and Lemma 2.6, it will be sufficient to show that $\mathscr{I}_{0}$-associated with $\zeta^{*}(V)$-contains no chain of length $r+1$.

Fix $v \in V$ with $S=S(v) \in \zeta^{*}(V)$.

Suppose for contradiction, $A_{0} \subset A_{1} \subset \cdots \subset A_{r} \subset A_{r+1}=\{1,2, \ldots, k\}$ is a chain in $\mathscr{I}_{0}$. Let $B_{0} \subset B_{1} \subset \cdots \subset B_{r+1}$ be the corresponding chain in $\mathscr{I}\left(S, \zeta^{*}(V)\right)$. Then for $i=0,1, \ldots, r$ there exists $v_{i} \in V$ with $s\left(v_{i}\right) \cap S=B_{i}$. By Dirichlet's principle there are two vectors, say $v_{i}, v_{j}, i<j$, such that $\left(v, v_{i}\right)$ and $\left(v, v_{j}\right)$ are in the same interval of length less than $m_{\text {inf }}^{2} / 2$.

Taking into consideration $\left|\beta_{\gamma}-\beta_{\gamma}^{\prime}\right|<\gamma$ and $\left(v, v_{j}\right)-\left(v, v_{i}\right)<m_{\text {inf }}^{2} / 2$, we infer

$$
\begin{aligned}
\frac{1}{2} m_{\mathrm{inf}}^{2} & >\left(v, v_{j}\right)-\left(v, v_{i}\right)=\sum_{\nu \in B_{i}} \alpha_{\nu}\left(\beta_{\nu}^{\prime}-\beta_{\nu}\right)+\sum_{\nu \in B_{j}-B_{i}} \alpha_{\nu} \beta_{\nu}^{\prime} \\
>-\gamma k \sqrt{l_{\text {sup }}}+m_{\mathrm{inf}}^{2} & \geqslant \frac{1}{2} m_{\mathrm{inf}}^{2} .
\end{aligned}
$$

A contradiction.

4. The proof of Proposition 1.2. In view of Corollary 2.3, it is sufficient to consider the case $L=L^{+}$, and show $m(k, k, M, L) \leqslant(\underset{k}{|L|+k})$. However this is a special case of Theorem 1.4.

5. Exterior products and covering points by hyperplanes. Denote by $P^{n}$ the real projective space of dimension $n$. A hyperplane is a subspace of dimension $n-1$.

THEOREM 5.1. Suppose $T \subset P^{n}, T=\left\{x_{1}, \ldots, x_{m}\right\}, s$ a positive integer and for every point $x_{i}$ there exist $s$ hyperplanes $H_{1}^{(i)}, \ldots, H_{s}^{(i)}$ so that $\left\{x_{i}, x_{i+1}, \ldots, x_{m}\right\} \cap\left(H_{1}^{(i)} \cup\right.$ $\left.\cdots \cup H_{s}^{(i)}\right)=\left\{x_{i+1}, \ldots, x_{m}\right\}$. Then $|T| \leqslant\left(\begin{array}{c}n+s \\ s\end{array}\right)$.

Proof. Let $\pi$ be a polarity of $P^{n}$. Then $\pi(T)=\left\{\pi\left(x_{i}\right): 1 \leqslant i \leqslant m\right\}$ is a collection of hyperplanes satisfying the following:

(i) For $1 \leqslant i \leqslant m$ there exist $s$ points $\left(\pi\left(H^{(i)}\right), \ldots, \pi\left(H_{s}^{(i)}\right)\right)$, none of which is on $\pi\left(x_{i}\right)$ so that $\pi\left(x_{j}\right)$ contains at least one of them for $j>i$.

If we replace in (i) the condition $j>i$ by $i \neq j$ then the upper bound $|T|=|\pi(T)|$ $\leqslant\left(\begin{array}{c}n+s \\ s\end{array}\right)$ is just Theorem 4.8 in [Lo]. A slight modification of Lovász's argument shows that the $s$-element sets $\left\{\left\{\pi\left(H_{1}^{(i)}\right), \ldots, \pi\left(H_{s}^{(i)}\right)\right\}: 1 \leqslant i \leqslant m\right\}$ which are points in the $s$ th symmetric power of $P^{n}$, are independent for $1 \leqslant i \leqslant m$. Consequently, their number $m$ does not exceed the dimension $\left(\begin{array}{c}n+s \\ s\end{array}\right)$.

Next we derive Theorem 1.4.

Arrange the vectors in $V$ so that $\left|v_{1}\right| \geqslant\left|v_{2}\right| \geqslant \cdots \geqslant\left|v_{m}\right|$. Then $\left(v_{i}, v_{i}\right)>\left(v_{i}, v_{j}\right)$ holds for $i<j \leqslant m$. Let $\lambda_{1}^{(i)}, \ldots, \lambda_{s}^{(i)}<\left(v_{i}, v_{i}\right)$ be such that $\left(v_{i}, v_{j}\right) \in\left\{\lambda_{1}^{(i)}, \ldots, \lambda_{s}^{(i)}\right\}$ holds for all $i<j \leqslant m$. Let us define

$$
G_{t}^{(i)}=\left\{v \in R^{n}:\left(v_{1}, v\right)=\lambda_{t}^{(i)}\right\}, \quad 1 \leqslant t \leqslant s .
$$


Then $G_{t}^{(i)}$ is a hyperplane in $R^{n}$. Define $H_{t}^{(i)}$ as the unique hyperplane in $P^{n}$ containing $G_{t}^{(i)}$.

Then $\left\{v_{1}, \ldots, v_{n}\right\}$ and the hyperplanes $H_{t}^{(i)}$ fulfill the assumptions of Theorem 5.1. Consequently, $|V|=m \leqslant\left(\begin{array}{c}n+s \\ s\end{array}\right)$.

As pointed out by the referee, Theorem 1.4 can be deduced also using the approach of Koornwinder [Ko].

6. Signed Steiner systems. For a set of vectors $V=\left\{v_{1}, \ldots, v_{m}\right\}$ define $-V=$ $\left\{-v_{1}, \ldots,-v_{m}\right\}$.

Proposition 6.1. If $L^{+}=L^{-}$then the following two conditions are equivalent:

(i) $V \in \mathscr{V}(n, k,\{ \pm 1\}, L)$,

(ii) $V \cup(-V) \in \mathscr{V}(n, k,\{ \pm 1\}, L \cup\{-k\})$.

COROLlaRY 6.2. If $-k \notin L, L^{+}=L^{-}$then

$$
2 m(n, k,\{ \pm 1\}, L)=m(n, k,\{ \pm 1\}, L \cup\{-k\})
$$

holds.

In view of Proposition 6.1 and Corollary 6.2, Theorem 1.5 will follow once we proved the next theorem.

TheOREM 6.3. Suppose $V \in \mathscr{V}(n, k,\{ \pm 1\},\{0, \pm 1, \ldots, \pm(t-1)\} \cup\{-k\})$.

Then

$$
|V| \leqslant \frac{\left(\begin{array}{c}
n \\
t
\end{array}\right)}{\left(\begin{array}{c}
k \\
t
\end{array}\right)} 2^{t}(k-t+1) .
$$

Moreover, for $k=t+1$ equality is possible if and only if there is an $(n, k, t)$ Steiner-system $\zeta$ so that $V$ consists of all $(0, \pm 1)$-vectors $v$ with $S(v) \in \zeta$.

Proof of Theorem 6.3. There are $2^{t}\left(\begin{array}{l}n \\ t\end{array}\right)(0, \pm 1)$-vectors with $t$ nonzero coordinates. For such a vector $w$ and $v \in \mathscr{V}$ define $w<v$ if $(w, v)=t$ (or equivalently if $v-w$ is a $(0, \pm 1)$-vector with $k-t$ nonzero entries). Define $V_{w}=\{v-w: w<v \in$ $V\}$. Then $V_{w} \in \mathscr{V}(n-t, k-t,\{ \pm 1\},\{-1,-2, \ldots,-2 t+1\})$.

A theorem of Delsarte, Goethals and Seidel [DGS] yields $\left|V_{w}\right| \leqslant k-t+1$.

Since for each $v \in V$ there are $\left(\begin{array}{l}k \\ t\end{array}\right)$ choices of $w, w<v,(6.1)$ follows.

Suppose now equality holds in (6.1), $k=t+1$. By our argument, $\left|V_{w}\right|=2$ must hold for all $w$. Suppose $w<v, w<v^{\prime}$. Since $\left(v, v^{\prime}\right) \leqslant k-2<t$, we infer $S(v)=$ $S\left(v^{\prime}\right)$, and $v$ and $v^{\prime}$ have opposite sign in $S(v)-S(w)$. Set $S=S(v)$.

We claim that all $(0, \pm 1)$-vectors $u$ with $S(u)=S$ are in $V$. Indeed, the contrary implies the existence of two $(0, \pm 1)$-vectors $u_{1}, u_{2}$ with $S\left(u_{1}\right)=S\left(u_{2}\right)=S, u_{1} \in V$, $u_{2} \notin V$ and $u_{1}$ and $u_{2}$ differ in only one position (i.e. $\left.\left(u_{1}, u_{2}\right)=k-2\right)$ ). Let $w_{1}$ be the unique $(0, \pm 1)$-vector with $t=k-1$ nonzero entries satisfying $w_{1}<u_{1}, w_{1}<u_{2}$. The equality in (6.1), as we have shown above, implies, via $\left|V_{w_{1}}\right|=2$, that $u_{2} \in V$, a contradiction. 
Now consider $\zeta=\{S(v): v \in V\}$. By the above we have $|V|=2^{k}|\zeta|$, that is $|\zeta|=\left(\begin{array}{c}n \\ k-1\end{array}\right) /\left(\begin{array}{c}k \\ k-1\end{array}\right)$. Clearly $\left|S \cap S^{\prime}\right| \leqslant k-2$ holds for $S, S^{\prime} \in \zeta$, thus $\zeta$ is a $(n, k, k-$ 1) Steiner-system and the statement follows.

7. Equidistant sets. In the case $M=\{ \pm 1\}, L=\{l\}$ one can get tight bounds. For vectors $w, v$ define $w \leqslant v$ if $w$ and $v$ coincide in each nonzero coordinate of $w$.

THEOREM [DF2]. Suppose $k>l \geqslant 1, V \in \mathscr{V}(n, k,\{ \pm 1\},\{l\})$ and $|V|>$ $\max \left\{(k-l)^{2}+(k-l)+1,(k-l)(l+2)\right\}$. Then there exists a $(0, \pm 1)$-vector $w$ with l nonzero positions so that $w<v$ holds for all $v \in V$ (and consequently the vectors $\{v-w: v \in V\}$ are pairwise orthogonal).

The corresponding theorem for $M=\{1\}$ was proved in [De].

\section{REFERENCES}

[BBS] E. Bannai, E. Bannai and D. Stanton, An upper bound for the cardinality of an s-distance set in real Euclidean space, Combinatorica 3 (1983), 147-152.

[B1] A. Blokhuis, Few-distance sets, CWI Tract 7 (1984).

[DGS] Ph. Delsarte, J.-M. Goethals and J. J. Seidal, Spherical codes and designs, Geom. Dedicata 6 (1977), 363-388.

[De] M. Deza, Une propriété extrémale des plans projectifs finis dans une classe de codes équidistants, Discrete Math. 6 (1973), 343-352.

[DF1] M. Deza and P. Frankl, On t-distance sets of $(0, \pm 1)$-vectors, Geom. Dedicata 14 (1983), 293-301.

[DF2] Every large set of equidistant $(0,+1,-1)$-vectors forms a sunflower, Combinatorica 1 (1981), 225-231.

[Fr] P. Frankl, On large vector systems with equal scalar products, Europ. J. Combinatorics 2 (1980), 123-125.

[FrW] P. Frankl and R. M. Wilson, Intersection theorems with geometric consequences, Combinatorica 1 (1981), 357-368.

[Fü] Z. Füredi, On finite set systems in which every intersection is a kernel of a star, Discrete Math. 47 (1983), 129-132.

[GRS] R. L. Graham, B. L. Rothschild and J. H. Spencer, Ramsey theory, Wiley, New York, 1980.

[Ko] T. Koornwinder, A note on the absolute bound for systems of lines, Proc. Koninklijke Nederl. Akad. Wet. 79 (1976), 152-153.

[Lo] L. Lovász, Flats in matroids and geometric graphs, Comb. Surveys: Proc. of 6th British Comb. Conference (P. J. Cameron, ed.), Academic Press, New York, 1977, pp. 45-87.

[RW] D. K. Ray-Chaudhuri and R. M. Wilson, On t-designs, Osaka J. Math. 12 (1975), 737-744.

[Rö] V. Rödl, A packing and covering problem, Europ. J. Combinatorics 6 (1986) (to appear).

Departement de Mathematiques, Universite de Paris ViI, CNRS, 2 Place Jussieu, 75005 Paris, FRANCE 